\title{
EFECTO DE RASTROJOS EN EL NITRÓGENO DE BIOMASA MICROBIANA EN UN AGROECOSISTEMA ARROCERO INUNDADO 1
}

\author{
Rodolfo Quirós-Herrera ${ }^{2}$, Carlos Ramírez-Martínez ${ }^{3}$
}

\begin{abstract}
RESUMEN
Efecto de rastrojos en el nitrógeno de biomasa microbiana en un agroecosistema arrocero inundado. Del ano 2002 al 2004 se investigó en un agroecosistema arrocero inundado a fin de evaluar cambios en la actividad de la biomasa microbiana y su contenido de nitrógeno "N", durante cuatro ciclos consecutivos del cultivo. Se comparó la tendencia del contenido de nitrógeno de la biomasa microbiana "NBM" en los sistemas siembra directa sobre rastrojos "SDR" y labranza mecanizada convencional "LMC". Durante el tercer ciclo de arroz se evaluó el efecto de los rastrojos de mucuna "Mucuna deeringiana (Bort.) Merr"; se comparó la materia orgánica del suelo "MOS" y el NBM. Adicionalmente, durante el cuarto ciclo de arroz del estudio, fueron contrastados cambios en la actividad de la biomasa microbiana en tres diferentes etapas de crecimiento del cultivo. En los primeros $5 \mathrm{~cm}$ del perfil del suelo la cantidad de NBM siempre fue mayor en SDR. En esta capa del suelo, la combinación de rastrojos de arroz acumulados durante tres ciclos con rastrojos de mucuna del ciclo previo, permitió una mayor estratificación de la MOS. Se observó un incremento de la MOS de 2,3 a 4,1\%, mientras que el NBM fue casi cuatro veces mayor. La respiración de los microorganismos del suelo y la mineralización del $\mathrm{N}$ fue afectada principalmente por la presencia continua de una lámina de agua sobre el suelo. Se concluyó que la SDR es un sistema capaz de complementar la fertilización química e incrementar los rendimientos del arroz mediante la gradual liberación de $\mathrm{N}$ y otros nutrimentos derivados de la descomposición de los rastrojos.
\end{abstract}

Palabras clave: Rastrojos de arroz, biomasa microbiana del suelo, siembra directa, nitrógeno, mucuna.

\begin{abstract}
Rice stubble and changes in nitrogen microbial biomass in a flooded rice agroecosystem. From 2002 to 2004, a research was carried out in a flooded rice agroecosystem in order to evaluate changes in the microbial biomass activity and its nitrogen content $(\mathrm{N})$, during four consecutive crop cycles. The trends of nitrogen content of the microbial biomass (NMB) in direct seeding into stubble (DSS) and conventional mechanized tillage (CMT) were compared. During the third rice cycle, the effect of mucuna (Mucuna deeringiana (Bort.) Merr) stubble was assessed, comparing the soil organic matter (SOM) and NMB. In addition, changes in the activity of microbial biomass through the growth stages of the rice crop during the fourth cycle were compared. Within the first $5 \mathrm{~cm}$ of the soil profile the amount of NBM was always greater for DSS. In this soil layer, the combination of rice stubbles accumulated during 3 cycles with mucuna stubbles from the previous cycle allowed a greater stratification of SOM. An increment from 2.3 to $4.1 \%$ was observed for SOM, while NBM quadrupled. Soil microorganism's respiration and $\mathrm{N}$ mineralization were primarily affected by the continuous presence of a water layer over the soil. It was concluded that SDR is a system capable of complementing the chemical fertilization and increasing rice yields through the gradual release of $\mathrm{N}$ and other nutriments from stubble decomposition.
\end{abstract}

Key words: Rice stubble, microbial biomass, direct seeding into stubble, nitrogen mineralization, respiration.

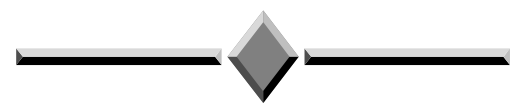

\footnotetext{
1 Recibido: 28 de marzo, 2005. Aceptado: 18 de julio, 2006. Parte de la tesis del primer autor. Facultad de Ciencias Agroalimentarias, Universidad de Costa Rica.

2 Estudiante programa de doctorado en sistemas de producción agrícola tropical sostenible "SPDATS", Facultad de Ciencias Agroalimentarias, Universidad de Costa Rica. Correo electrónico: rodolfo.quiros@costarricense.cr

3 Correo electrónico: carlosm20@gmail.com
} 


\section{INTRODUCCIÓN}

A pesar de que algunos cultivos pueden crecer hasta su madurez en soluciones de nutrimentos, la mayoría de las plantas terrestres se establecen y fructifican cultivadas sobre el suelo. Generalmente el suelo no provee todas las condiciones necesarias para el óptimo desarrollo de los cultivos y es necesario realizar aportes de fertilizantes que permitan mejorar la posibilidad de obtener altos rendimientos. La deficiencia de nutrimentos esenciales o la presencia de éstos en cantidades limitadas, reduce la masa de raíces y tallos producidos por los cultivos durante su desarrollo. El efecto de los nutrimentos, tanto en el crecimiento vegetativo como reproductivo de las plantas, y particularmente del nitrógeno " $\mathrm{N}$ " es mayor a medida que concentraciones apropiadas de éstos sean alcanzables por las raíces del cultivo.

Según Norman et al. (2003), de los macronutrimentos tomados del suelo el $\mathrm{N}$ es el más importante en el crecimiento del arroz (Oryza sativa L.). Generalmente el $\mathrm{N}$ es absorbido en grandes cantidades por las plantas de arroz en forma de amonio " $\mathrm{NH}_{4}{ }^{+}$" y nitrato " $\mathrm{NO}_{3}{ }^{-"}$, y es de vital importancia para la productividad del cultivo. Su concentración media en la planta es de 1,$34 ; 1,8$ y $0,49 \%$ en granos, hojas y tallos respectivamente (Anders et al. 2004). El amonio y nitrato disueltos en agua constituyen la fracción más importante del $\mathrm{N}$ disponible en la solución del suelo. Su concentración puede ser modificada principalmente cuando: 1) hay aportes debidos a la mineralización de la materia orgánica del suelo (MOS) por acción de microorganismos, 2) se aplican fertilizantes nitrogenados, 3) el N es inmovilizado por la biomasa microbiana del suelo "BMS", 4) al ser absorbido por las plantas, 5) incrementa o decrece la cantidad de agua en el suelo.

El $\mathrm{N}$ prácticamente se halla ausente del material original de los suelos, y excluyendo la fertilización nitrogenada, su presencia se debe fundamentalmente a la actividad biológica de los microorganismos del suelo. Hay indicios de fijación biológica no simbiótica de $\mathrm{N}$ asociada a la descomposición de los rastrojos del arroz (Yamaguchi 1979). Cuando ocurre la descomposición de los rastrojos y se mineraliza la MOS se libera $\mathrm{N} \mathrm{y}$ otros nutrimentos, lo cual favorece una nutrición más balanceada del arroz. Debido a que los microorganismos del suelo forman parte integral de la MOS, su actividad puede aportar $\mathrm{N}$ en forma natural y más sostenible que la fertilización nitrogenada.
La MOS sirve como fuente de energía y nutrimentos para microorganismos del suelo como bacterias, hongos, protozoarios y nemátodos (Doran y Parkin 1994). Éstos junto con macroorganismos y raíces de plantas forman la fracción viviente de la MOS, y en general constituyen más del $70 \%$ de la misma (Theng 1987). Los microorganismos del suelo contienen el $3 \%$ del $\mathrm{N}$ almacenado en los ecosistemas terrestres y su actividad heterotrófica en los suelos es responsable de la mineralización de la MOS, proceso que en forma natural libera $\mathrm{NH}_{4}{ }^{+}$a la solución del suelo (Zak et al. 1994). Más del $50 \%$ del $N$ utilizado por el arroz inundado puede ser derivado de la mineralización de la MOS por bacterias (IRRI 1990).

Aunque en forma temporal los microorganismos del suelo compiten con el cultivo al consumir e inmovilizar el $\mathrm{N}$ y otros nutrimentos, después de que tales seres vivientes completan su ciclo vital y mueren, mediante la descomposición de sus tejidos liberan a la solución del suelo los nutrimentos antes inmovilizados, en formas disponibles para las plantas. En adición al suministro de $\mathrm{N}$ y otros nutrimentos, la MOS contribuye a estabilizar los agregados y mantener abiertos los macroporos necesarios para la infiltración del agua, lo cual ayuda a reducir la escorrentía y la erosión. A pesar de que estas razones no son siempre conocidas, generalmente los suelos son más fáciles de manejar cuando el contenido de MOS es alto, especialmente en las zonas tropicales (Johnson 1991).

La biomasa microbiana del suelo (BMS) es una fracción de la MOS que puede afectar significativamente la fertilidad del suelo, pues controla fuertemente la mineralización de la MOS y la tasa de liberación de N, por este motivo constituye a la vez una fuente y sumidero de éste y otros nutrimentos (Fornasier et al. 2002). Por las razones mencionadas, el $\mathrm{N}$ contenido en la BMS constituye un indicador importante en el estudio de la fertilidad del suelo. En cualquier terreno la dinámica de los componentes bióticos es afectada por factores ambientales tales como; características del suelo, la temperatura, y formas de manejo de los rastrojos. Cuando a través del laboreo y la incorporación de los rastrojos dentro del perfil del suelo se disturba el equilibrio biológico, se ocasionan cambios en la composición y actividad de la BMS, con distintas implicaciones en su fertilidad en el corto y largo plazo (Leita et al. 1999).

El sistema de labranza del suelo afecta la velocidad de la descomposición de la MOS (Etana et al. 
1999). La labranza ocasiona disturbios al suelo y una mayor tasa de descomposición de los rastrojos en comparación con terrenos sin labrar. La continua labranza del suelo conduce a menor nivel de agregación y estabilidad física de la MOS (Carter et al. 1998). Con la preparación física del suelo en la labranza mecanizada convencional (LMC) se incrementa de manera efímera la biomasa microbiana, produciendo mayor cantidad de reacciones concurrentes de inmovilización, mineralización y estabilización (Voroney et al. 1989). La oxidación biológica en conjunto con la erosión puede ocasionar pérdidas de MOS equivalentes a la mitad de la fuente natural (Tiessen et al. 1994). El rápido agotamiento de la MOS podría afectar negativamente la calidad del suelo y reducir la productividad del cultivo (Bayer et al. 2001).

Las frecuentes alteraciones del suelo provocadas por la labranza en LMC, hacen que las tasas de descomposición de los rastrojos y la mineralización de la MOS sean generalmente más rápidas que en SDR pero de corta duración. En LMC los rastrojos y la MOS son mezclados y distribuidos de manera homogénea dentro de todo el perfil del suelo afectado por la labranza. Los procesos de descomposición en este caso son dominados por bacterias, las cuales tienen menor eficiencia en la asimilación de nutrimentos que los hongos (Gale y Cambardella 2000). Se presume que en corto tiempo las reservas de MOS son consumidas por las bacterias y si los productos de su descomposición no son oportunamente aprovechados por el cultivo, pueden perderse del agroecosistema.

Debido a que en el sistema de siembra directa sobre rastrojos (SDR) la preparación física del suelo es mínima y mayor cantidad de residuos vegetales permanece en la superficie, la descomposición de la MOS es más lenta que en LMC. La reducción en la intensidad de la labranza y el uso de sistemas de cultivo que maximicen la adición de residuos a la superficie del terreno, han sido prácticas eficientes para mantener o incrementar la MOS (Bayer et al. 2001). A medida que transcurren más ciclos de cultivo en SDR, se produce una mayor concentración de MOS en la capa del suelo más cercana a la superficie, mientras que en LMC ésta se distribuye de manera uniforme en todo el perfil afectado por la labranza. El grado de estratificación de la MOS en el perfil puede indicar la calidad del suelo debido a que su acumulación en la superficie es esencial para controlar la erosión, incrementar la infiltración del agua y regular la disponibilidad de nutrimentos (Franzluebbers 2002).

Cuando las condiciones ambientales son adecuadas, la actividad de los microorganismos del suelo y el potencial para la mineralización de la MOS son mayores en el horizonte superficial, en comparación con el resto del perfil. Conforme se produce la acumulación de los rastrojos en la superficie, los microorganismos tienen a disposición más sustrato para actuar, y a través de su descomposición, progresivamente van incrementando la fracción orgánica del suelo, con una marcada estratificación en las capas de suelo menos profundas. Los rastrojos superficiales son descompuestos principalmente por hongos, los cuales tienen una alta eficiencia de asimilación de $\mathrm{N}$ y otros nutrimientos (Gale y Cambardella 2000). Una mayor concentración de MOS cerca de la superficie, permite en un proceso continuo de mineralización, mayor cantidad de NBN en SDR con respecto a LMC.

Otro factor determinante a considerar en la actividad de la BMS en agroecosistemas cultivados con arroz es el efecto del agua, de manera particular en terrenos bajo inundación con lámina de agua continua. En ambientes de este tipo un decrecimiento de la capacidad natural del suelo para suplir $\mathrm{N}$ podría reducir la productividad del arroz bajo inundación en forma intensiva y continua (Cassman et al. 1995). En los agroecosistemas arroceros que permanecen inundados durante la mayor parte del cultivo, la condición anaeróbica predominante hace que las poblaciones y la actividad microbiana sean diferentes a cultivos de arroz en secano, y posiblemente con impactos distintos en la disponibilidad del $\mathrm{N}$ en el suelo.

En arroz inundado el suelo se mantiene bajo una lámina de agua continua desde el momento en que las plántulas de arroz han completado su emergencia hasta dos semanas antes de la cosecha. En ese lapso la ausencia de oxígeno en el suelo restringe la actividad de microorganismos aeróbicos y la descomposición de los rastrojos es más lenta, posiblemente porque sólo pueden mantenerse activos los anaeróbicos y los aeróbicos facultativos. Debido a que en la región del estudio las temperaturas generalmente se mantienen altas $\left(28^{\circ} \mathrm{C}\right)$, con muy leves variaciones durante el ano, éstas normalmente son favorables para la descomposición de rastrojos y por tanto no parecen ser un factor limitante para la actividad microbiana. 
Desde la cosecha del arroz hasta la siembra del ciclo siguiente el terreno generalmente se mantiene drenado. En condiciones normales la humedad del suelo únicamente corresponde al remanente del cultivo anterior y a la aportada por las lluvias. Si la humedad es adecuada, los procesos de descomposición de rastrojos y mineralización de la MOS podrían incrementarse durante ese período, porque en condiciones predominantemente aeróbicas la actividad de los microorganismos aeróbicos es mayor. Una manera de optimizar la descomposición de los rastrojos del arroz es aprovechar el lapso de descanso entre ciclos del cultivo, cuando los terrenos no son utilizados con fines productivos, intercalando la siembra de una planta leguminosa a manera de abono verde.

Los métodos tradicionales para cuantificar la MOS no permiten estimar en forma adecuada la cantidad de nutrimentos que se produce con su mineralización ni cuánto de éstos son directamente aprovechados por el cultivo (Theng 1987). La MOS consiste en un complejo de compuestos, con fracciones vivientes (microorganismos, raíces de plantas, macroorganismos) y no vivientes (humus y macromateria orgánica). Sin embargo, las fracciones más lábiles, particularmente la BMS, constituye un mejor indicador de los efectos de las prácticas de labranza y la lámina de agua en las transformaciones de la MOS.

Puesto que una proporción significativa del $\mathrm{N}$ disponible en el suelo se deriva de la mineralización de la MOS (Cabrera et al. 1994), conocer la cantidad de N mineralizable es importante para determinar la dosis de fertilizante que optimiza los rendimientos del cultivo, sin incrementar la alteración ambiental por pérdidas de este elemento y la consecuente contaminación de suelos y aguas. Varios estudios indican que en la mayoría de los suelos la MOS se correlaciona con la BMS (Anderson y Domsch 1989; Wardle 1992; Meléndez 1997; Salas y Ramírez 1999). En consecuencia, a través de mediciones de BMS se puede inferir su nivel de actividad y estimar la intensidad a la cual la MOS está siendo mineralizada (Uribe 1999).

Por otra parte, varias especies leguminosas son usadas como cultivos de cobertura o abonos verdes para mejorar la fertilidad del suelo e incrementar la producción del arroz (Palaniappan y Siddeswaran 1999). La mucuna “Mucuna deeringiana (Bort.) Merr." es una especie leguminosa que permite incrementar los rendimientos del arroz en un 25\%. Esta leguminosa florece entre 45 y 50 días después de la siembra y produce hasta 5 t/ha de rastrojos (Singh et al. 1995). En campos arroceros inundados, el cultivo de leguminosas como abonos verdes puede efectuarse durante los períodos cuando no se siembra arroz, en lapsos de descanso del terreno entre la cosecha de un ciclo y la siembra del siguiente ciclo arrocero. La siembra de mucuna como abono verde, intercalada entre dos ciclos del cultivo comercial, mejora la descomposición de los rastrojos del arroz, e incrementa el $\mathrm{N}$ disponible en el suelo derivado de la mineralización de la materia orgánica (Ladha et al. 2000). Esto conduce a una mejor calidad productiva del terreno, lo cual permite elevar la eficiencia en la fertilización nitrogenada y mayores rendimientos en la cosecha del arroz.

Si se admite que el efecto de los rastrojos de arroz que quedan acumulados sobre la superficie del terreno en SDR es distinto a cuando éstos son incorporados dentro del perfil del suelo, tal como ocurre en LMC, entonces al mantener los rastrojos sobre el suelo en vez de incorporarlos dentro el perfil, se lograría una tasa de descomposición más lenta, que permitiría un flujo más regulado de la liberación del $\mathrm{N}$ orgánico en el suelo. Una forma de comprobar esta hipótesis consiste en investigar si existen diferencias significativas en la tasa de descomposición de los rastrojos y la mineralización de la MOS entre los sistemas de preparación del terreno para el cultivo SDR y LMC. La tasa de descomposición de los rastrojos se regularía por efecto de la labranza en LMC y la ausencia de ésta en SDR.

Con el objetivo de evaluar el efecto de la labranza y los rastrojos de arroz en los sistemas mencionados, en este estudio se determinó la cantidad de nitrógeno de la biomasa microbiana (NBM) durante cuatro ciclos consecutivos de arroz inundado. Además se evaluó el efecto en la descomposición de los rastrojos de arroz acumulados durante tres ciclos de cultivo previos, al ser combinados rastrojos de mucuna, una leguminosa con capacidad de fijación biológica de $\mathrm{N}$ atmosférico. Para esto se determinó el contenido de MOS y NBM antes y después de la siembra de mucuna. Adicionalmente, durante el último ciclo de arroz en estudio, a través de determinaciones de mineralización del $\mathrm{N}$ y respiración de la BMS, se evaluaron cambios en la actividad de los microorganismos del suelo en tres distintas etapas de desarrollo del arroz. 


\section{MATERIALES Y MÉTODOS}

\section{Características del sitio en estudio}

La etapa experimental del estudio se desarrolló entre enero del 2002 y mayo del 2004, en un agroecosistema arrocero de la hacienda el Pelón de la Bajura, en la Región Pacífico Norte de Costa Rica. El sitio corresponde a la zona de vida bosque húmedo premontano transición basal tropical y presenta topografía plana, con elevaciones medias de 10 a $20 \mathrm{msnm}$, ubicado entre las coordenadas verticales 381-384 y 267-274 horizontales de la hoja Tempisque ${ }^{4}$. Según datos de la estación hidrometeorológica local ${ }^{5}$, la temperatura media anual presentó pequenas fluctuaciones, con una media de $27,6{ }^{\circ} \mathrm{C}$ y valores mínimos y máximos respectivos de 22,9 a $34,7{ }^{\circ} \mathrm{C}$; la precipitación media fue de $1.722 \mathrm{~mm} / \mathrm{ano}$, con el $66 \%$ de las lluvias concentradas en junio, setiembre y octubre; la humedad relativa varió entre 61 y $90 \%$. La textura del suelo en el área del experimento fue francoarcillosa (33\% arena, 29\% limo, 38\% arcilla), con color gris oscuro a negro en el perfil estudiado. El pH del suelo fue de 6,5 y la capacidad de intercambio catiónico (CICE) de $22,2 \mathrm{cmol}(+)^{6}$.

\section{Arreglo experimental}

El experimento se realizó en un lote arrocero cultivado a escala comercial con arroz bajo inundación con lámina de agua continua, identificado como Playitas. En este lote se instaló un par de parcelas permanentes cuadradas con $4.900 \mathrm{~m}^{2}$ de superficie cada una, separadas entre sí por bordes de 10 metros de ancho. El arreglo experimental consistió en parcelas contiguas asignando los sistemas SDR y LMC a cada parcela, con cinco repeticiones por lote. Las parcelas fueron divididas en 49 subparcelas cuadradas con $100 \mathrm{~m}^{2}$ de área efectiva cada una, y en forma aleatoria se seleccionaron cinco subparcelas por sistema de siembra para efectuar los respectivos muestreos de suelo.

\footnotetext{
4 Instituto Geográfico Nacional. 1973. Mapa básico de Costa Rica, Hoja Tempisque (3147-III), escala 1:50,000.

5 Estación hidrometeorológica instalada en la Hacienda El Pelón de la Bajura, Bagaces, Guanacaste.

6 Análisis realizados en el Laboratorio de Suelos del Centro de Investigaciones Agronómicas. UCR.
}

\section{Manejo del arroz}

En la cosecha del arroz se usó una cosechadora con secciones articuladas, capaces de desprender los granos del arroz sin cortar los tallos, las cuales dejaron en forma casi intactas las canas en pie y la mayor parte del follaje. Sólo la granza (cascarilla del arroz) y pequenos trozos de hojas o tallos fueron retirados del sitio por la cosechadora. Mediante muestreo y determinación del peso de la biomasa seca aérea, se estimó la cantidad de rastrojos de malezas y de arroz que quedaron sobre el terreno al final de cada ciclo de cultivo. Según el método de línea transepto (Shelton et al. 2000) la cobertura del suelo lograda por los rastrojos en todos los casos evaluados fue mayor al $60 \%$. La biomasa de raíces (rastrojos subsuperficiales) no fue considerada en este estudio debido a la dificultad para manipular suelos de textura pesada.

Para controlar el crecimiento de la vegetación y los rastrojos, en las parcelas bajo SDR en cada ciclo del estudio se efectuó una aplicación total de herbicida sistémico postemergente, cinco días, antes de la siembra. Se aplicó glifosato a razón $3 \mathrm{~kg} / \mathrm{ha}$ de producto formulado $68 \mathrm{SG}$ en 200 1/ha de agua. El cultivo fue sembrado manteniendo el total de rastrojos de arroz remanentes de la cosecha previa junto con las malezas desecadas por el tratamiento herbicida. En sitios tratados con LMC el suelo fue preparado mediante una pasada de rastra rompedora, una de rastra afinadora y finalmente un pase de rodillo compactador. Para incorporar completamente los rastrojos se empleó una rastra con 40 discos de $98 \mathrm{~cm}$ de diámetro. En la superficie del terreno no quedó en forma visible restos de rastrojos ni malezas.

En los ciclos evaluados se utilizó una sembradora SEMEATO TDNG 420, calibrada para sembrar 180 $\mathrm{kg} / \mathrm{ha}$ de semilla certificada de arroz. Para activar la germinación del cultivo se aplicó riego intermitente durante tres a cuatro semanas después de la siembra. $\mathrm{Al}$ inicio del ensayo los terrenos fueron micro nivelados para asegurar uniformidad en la cobertura con una lámina de agua de $10 \mathrm{~cm}$ de altura, la cual se mantuvo en forma continua una vez que las plántulas alcanzaron el estado de cuatro hojas. De acuerdo al programa de fertilización de la finca, para asegurar un adecuado suministro de fósforo, potasio y magnesio, al momento de la siembra se fertilizó con 50,125 y $8 \mathrm{~kg} / \mathrm{ha}$ de súper triple fosfato, sulfato de potasio y óxido de magnesio respectivamente. 


\section{Manejo de mucuna}

La mucuna se cultivó entre los meses de abril a julio, para lo cual se aprovechó el período entre las siembras de arroz de temporada seca y lluviosa, cuando normalmente el terreno se mantiene en descanso sin cultivo comercial. Después de la cosecha del arroz se realizó una aplicación total con herbicida glifosato, a razón de $3 \mathrm{~kg} / \mathrm{ha}$ de producto formulado $68 \mathrm{SG}$ en 200 1/ha de agua, a fin de controlar el crecimiento de rebrotes en la soca y en las áreas bajo LMC se efectuó la labranza del terreno. Tanto en LMC como en SDR se sembró mucuna en forma manual, a razón de 30 $\mathrm{kg} / \mathrm{ha}$, en este último caso bajo los rastrojos del arroz. Para promover la germinación de las semillas se efectuó mojes frecuentes del terreno inmediatamente después de la siembra. Sin aplicar fertilización química, se permitió el crecimiento de las plantas leguminosas hasta alcanzada la fase floración, a 120 dds (días después de la siembra). En ese momento se realizó otra aplicación de glifosato, a la misma dosis anterior, para controlar el crecimiento del cultivo leguminoso e inducir la descomposición de sus rastrojos. Mediante muestreo y determinación del peso de la biomasa seca aérea, se estimó el peso de los rastrojos de mucuna en $690 \mathrm{~kg} / \mathrm{ha}$. El levantamiento de muestras en el campo se efectuó a 30 días después de iniciada la descomposición de los rastrojos de mucuna.

\section{Muestreo del suelo}

Al inicio de cada ciclo de cultivo, cinco días antes de introducir la lámina de agua permanente al arrozal, se tomó muestras compuestas de suelo distribuidas al azar, en cinco puntos de muestreo por subparcela, entre 0 y $5 \mathrm{~cm}$ de profundidad. Debido a que en SDR el contenido de MOS se concentra en la capa superficial del suelo, se consideró que en el ámbito de profundidad elegido en el presente estudio es donde existe mayor posibilidad de observar asociaciones entre las variables a evaluar. Cada muestra compuesta con un peso aproximado de $500 \mathrm{~g}$ se empacó en bolsas plásticas debidamente identificadas y se envió inmediatamente al laboratorio, para los respectivos análisis. Durante el cuarto ciclo del estudio fueron efectuados muestreos complementarios al momento de la siembra, floración y antes de la cosecha del arroz. El primer muestreo se realizó con el terreno húmedo a capacidad de campo, mientras que en los últimos dos las muestras fueron tomadas con el suelo cubierto por la lámina de agua.

\section{Características evaluadas}

Durante los cuatro ciclos consecutivos de cultivo que abarcó el estudio se evaluó el contenido de MOS (\%) y el nitrógeno de la biomasa microbiana NBM $(\mathrm{mgN} / \mathrm{kg}$ de suelo). Adicionalmente, en el último ciclo evaluado se determinó la mineralización de nitrógeno $(\mathrm{MN})(\mathrm{mgN} / \mathrm{kg}$ suelo día) y la respiración de la biomasa microbiana (RBM) ( $\mathrm{mgC} / \mathrm{kg}$ suelo día). Para determinar el NBM se empleó el método de "Fumigación - extracción" en el cual las muestras de suelo son expuestas a vapores de cloroformo para destruir las membranas de los microorganismos y liberar al medio los contenidos celulares de N, para ser extraído y evaluado. La técnica de respiración fue utilizada en este estudio como variable indicadora de la actividad total de los microorganismos del suelo (Uribe 1999). Los análisis de laboratorio fueron efectuados en el Centro de Investigaciones Agronómicas de la Universidad de Costa Rica (UCR). Con los datos obtenidos se efectuó pruebas de comparación de medias entre sistemas de siembra y fechas de muestreo, así como análisis de correlación entre las respectivas variables.

\section{RESULTADOS Y DISCUSIÓN}

Los resultados son presentados en tres secciones correspondientes a los principales objetivos planteados en el estudio. En la primera sección se presenta la variación en la cantidad de NBM, según el sistema de labranza utilizado y el momento de muestreo. También se contrasta los cambios observados en el contenido de MOS y NBM por efecto de los rastrojos de una siembra de mucuna entre dos ciclos de cultivo del arroz. En las últimas dos secciones se compara las tasas de mineralización de nitrógeno y respiración de la BM del suelo, durante las fases de siembra, floración y precosecha del cultivo.

\section{Cambios en la cantidad de biomasa microbiana de nitrógeno}

La cantidad de NBM determinada en la capa de suelo comprendida entre 0 y $5 \mathrm{~cm}$ de profundidad, presentó variaciones que fluctuaron entre 12 y $32 \mathrm{~kg}$ / ha durante el período evaluado (Figura 1). Las determinaciones 


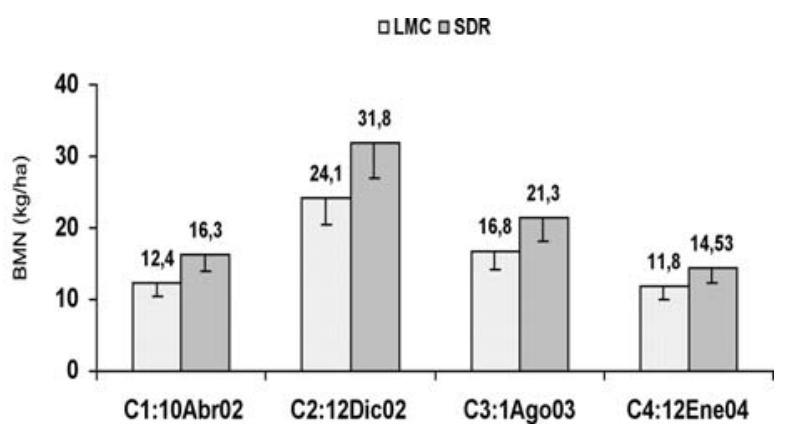

Figura 1. Cambios en el contenido de nitrógeno de la biomasa microbiana "NBM" según sistema de labranza (siembra directa sobre rastrojos "SDR" y labranza mecanizada convencional "LMC"), durante cuatro ciclos consecutivos de arroz inundado. Lote Playitas, Guanacaste, Costa Rica. 2002 - 2004.

de NBM se consideran una medida momentánea que puede variar en respuesta a diversos factores (Bertsh 2003). Las fluctuaciones pueden deberse al efecto de las lluvias y a la alternancia de períodos secos y húmedos característicos del régimen de riego en el cultivo de arroz inundado. Puesto que la descomposición de los rastrojos y la mineralización de la MOS por los microorganismos del suelo requieren apropiadas condiciones de aireación, humedad y temperatura, según su tipo; los frecuentes cambios ambientales entre temporadas secas y períodos bajo inundación influyen en la actividad de los microorganismos del suelo y por ende la cantidad de derivados intermedios como el NBM.

Aunque ambos sistemas de labranza mostraron patrones similares de variación en los diferentes momentos de muestreo realizados durante cada uno de los ciclos de cultivo que abarcó el experimento, la cantidad de NBM en SDR fue significativamente diferente $(\mathrm{P} \leq 0,05)$ a la de LMC, el promedio fue $29 \%$ mayor. Los mayores valores de NBM fueron observados en los ciclos dos y tres, cuando en el período comprendido entre la cosecha del ciclo previo y la fecha de muestreo el suelo se mantuvo húmedo por la ocurrencia de lluvias. Por el contrario, para los ciclos uno y cuatro dicho período coincidió con la temporada seca, y el suelo permaneció con déficit de humedad.

La biomasa microbiana forma parte de la fracción orgánica del suelo, es un agente determinante en la descomposición de la materia orgánica y controla la tasa a la cual los nutrimentos son transformados para quedar disponibles o ser absorbidos por el cultivo (Castro 1995; Uribe 1999). En ambos sistemas el NBM mostró alta correlación con el contenido de MOS, con coeficientes de determinación $\left(\mathrm{R}^{2}\right)$ de 0,79 y 0,86 respectivamente para LMC y SDR. Esto confirma la utilidad del NBM como variable indicadora de la tasa a la cual la MOS está siendo mineralizada por los microorganismos del suelo, cuya actividad fluctúa según condiciones cambiantes del ambiente edáfico.

Las diferencias observadas en la cantidad de NBM posiblemente sean debidas a la distinta distribución de la MOS y a su influencia en la actividad de los microorganismos del suelo, según el sistema de labranza aplicado. En SDR la distribución del $\mathrm{N}$ está altamente afectada por la acumulación de rastrojos en la superficie del terreno y las condiciones favorables para la actividad biológica cerca de la superficie (Doran 1980). En este sistema la MOS se mantiene o incrementa cuando se compara con LMC (Reeves 1997). Aunque el incremento de MOS en todo el perfil del suelo no es significativo, ocurre una mayor concentración en los primeros $5 \mathrm{~cm}$ de profundidad (Rasmussen y Collins 1991). Una mayor concentración de MOS en las capas del perfil próximas a la superficie representa más sustrato para la actividad de los microorganismos del suelo.

El manejo dado a los rastrojos es otro factor que pudo haber influido en la cantidad de NBM. Por sus altos contenidos de sílice y lignina la degradación de los rastrojos de arroz es más lenta que en otros cultivos de la misma familia. Mientras que en SDR los residuos vegetales se dejaron enteros y en su mayoría en pie, en LMC la labranza causó la ruptura de tallos y raíces, fragmentándolos e incorporándolos dentro del perfil, con lo cual quedaron expuestos a la rápida acción de los microorganismos del suelo. El corte de los residuos en partículas pequenas aumenta su superficie específica de contacto con el suelo, esto pudo incrementar la población microbiana y el intercambio de nutrientes y agua con el suelo circundante (Fruit et al. 1999). A medida que se reduce el tamano de los rastrojos su descomposición se incrementa (Angers y Recous 1997).

La mayor cantidad de NBM determinada durante todo el estudio se observó en SDR luego de sembrar mucuna (Mucuna deeringiana (Bort.) Merr.) entre dos ciclos de arroz, con lo cual se logró aprovechar la temporada en que no se siembra arroz (Figura 2). En 


\section{$\square$ MOS $\square$ NBM}

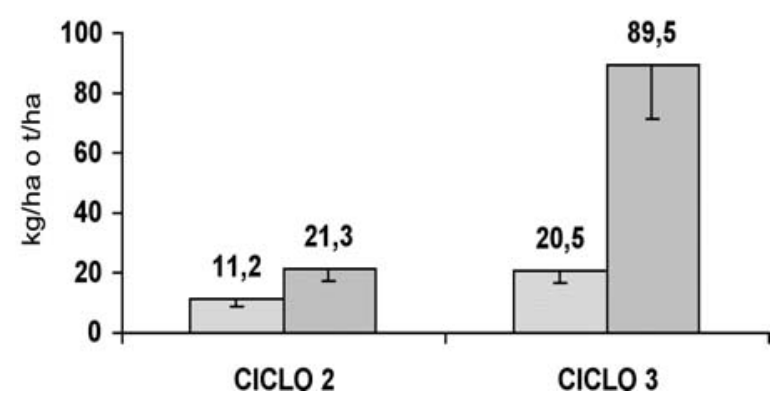

Figura 2. Contenido de materia orgánica del suelo "MOS" ( $\mathrm{t} / \mathrm{ha}$ ) y nitrógeno de la biomasa microbiana "NBM" $(\mathrm{kg} / \mathrm{ha})$ en los primeros $5 \mathrm{~cm}$ del perfil del suelo, en un terreno bajo siembra directa sobre rastrojos "SDR", antes y después de una siembra de mucuna. Lote Playitas, Guanacaste, Costa Rica. 2003.

este caso las plantas de mucuna junto con sus malezas asociadas fueron controladas con un tratamiento herbicida y sus rastrojos se sumaron a los del arroz del ciclo previo. La mucuna es una planta leguminosa con capacidad para realizar la fijación biológica del nitrógeno en los nódulos de sus raíces (Ferrera y Alarcón 2001).

En este caso, la combinación de rastrojos de arroz con los de la planta leguminosa permitió un cambio significativo en el contenido de MOS en los primeros cinco centímetros del perfil, el cual pasó de 2,24\% a 4,1\%. En el mismo lapso el NBM incrementó en cuatro veces la cantidad existente al inicio del ciclo previo. Esto muestra concordancia con lo determinado por Powlson y Brookes (1987) en el sentido de que las mediciones de biomasa microbiana dan una pronta indicación de cambios en el contenido de MOS. A mayor profundidad en el perfil no hubo diferencias significativas entre un ciclo y otro en ninguna de las dos variables.

Los datos sugieren que la descomposición combinada de rastrojos de arroz con los de mucuna produjo efecto sinérgico en las cantidades de MOS y NBM en SDR. Además de la fijación biológica de $\mathrm{N}$, la mucuna posee una baja relación $\mathrm{C}: \mathrm{N}$, que permite equilibrar la alta relación $\mathrm{C}: \mathrm{N}$ de los rastrojos de arroz. Posiblemente el balance neto que resulta de la combinación mejore la actividad de los microorganismos y con ésta la descomposición de los rastrojos así como la acumulación de MOS cerca de la superficie del terreno. Los anteriores resultados muestran la ventaja de mantener los rastrojos de arroz en la superficie, en vez de incorporarlos al perfil y; asímismo, queda sustentada la importancia de combinar los rastrojos de arroz con los de plantas leguminosas, a fin de mejorar la descomposición de los mismos e incrementar la disponibilidad de $\mathrm{N}$ para los cultivos siguientes.

Horwath y Kessel (1998) encontraron que una porción significativa del $\mathrm{N}$ procedente de la fertilización nitrogenada aplicada al arroz durante el ciclo previo fue encontrada en el NBM y en la MOS. Esto permite suponer que regular la descomposición de los rastrojos, de modo que se mantengan condiciones favorables para incrementar la actividad de los microorganismos, es una forma de evitar las altas pérdidas que ocurren con la fertilización nitrogenada, pues éstos conservan el $\mathrm{N}$ en una forma lábil en su biomasa. González y Ramírez (1991) afirman que los rastrojos de una planta leguminosa como la soya provocaron aumentos en la producción de arroz del orden de 1,5 t/ha, independientemente del nivel de fertilización nitrogenada aplicada al cultivo. Debido a que la biomasa microbiana controla la tasa a la cual el $\mathrm{N}$ está disponible para la absorción del cultivo, constituye una fuente natural de $\mathrm{N}$ que debe ser considerada como complemento de la fertilización con este nutrimento.

\section{Mineralización de N a partir de la MOS}

En la Figura 3 se presentan los cambios observados en la mineralización del nitrógeno (MN) durante

\section{口LMC $\square$ SDR}

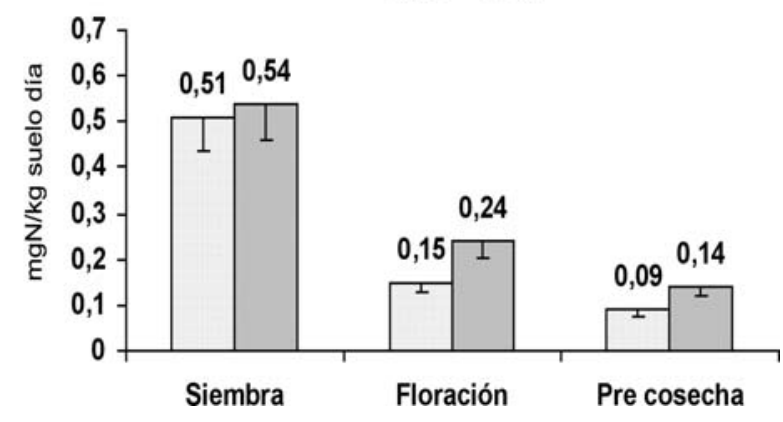

Figura 3. Mineralización de nitrógeno "N" a partir de la materia orgánica del suelo "MOS" en diferentes fases dentro del ciclo de desarrollo de un arrozal inundado. ("SDR", siembra directa sobre rastrojos, "LMC" labranza mecanizada convencional). Lote Playitas, Guanacaste, Costa Rica. 2004. 
tres etapas dentro del ciclo de desarrollo del cultivo. En todos los casos las medias correspondientes al sistema SDR fueron significativamente mayores $(\mathrm{P} \leq$ $0,05)$ a las de LMC. En ambos sistemas de labranza, las tasas más altas de $\mathrm{MN}$ se determinaron con las muestras obtenidas a los 10 días después de la siembra del arroz, el terreno húmedo a capacidad de campo. Éstas disminuyeron drásticamente en las otras dos fechas evaluadas, cuando el terreno estuvo bajo lámina de agua continua. La disminución en las tasas de MN durante la floración y antes de la cosecha del arroz probablemente fue causada por la lámina de agua sobre el suelo. Se presume que las condiciones anaeróbicas del arrozal inundado habrían impedido la actividad de los microorganismos aeróbicos, y de ese modo fueron más lentos los procesos involucrados en la mineralización tanto en SDR como en LMC.

Algunos autores sostienen que la $\mathrm{MN}$ en el suelo puede aportar hasta un 30\% del nitrógeno disponible que es asimilado por el cultivo (Harris y Hesterman 1990; Janzen y Schaalje 1992). La magnitud del aporte depende del tipo de rastrojos del ciclo previo, de su relación $\mathrm{C} / \mathrm{N}$ y de su manejo. En LMC la respuesta microbiana a la incorporación de los rastrojos generalmente es muy rápida, durante las dos semanas siguientes, pero declina substancialmente después (Lundquist et al. 1999). Desde un punto de vista práctico, esta liberación repentina del $\mathrm{N}$ de los rastrojos no permite satisfacer la demanda de $\mathrm{N}$ del arroz y hay alto riesgo de pérdidas por lixiviación y denitrificación durante lluvias tempranas o el riego (Bremer y Kessel 1992). Por el contrario, en SDR la mineralización del $\mathrm{N}$ ocurre de manera lenta y gradual, de tal modo que permite sincronizar sus aportes con la fertilización nitrogenada del arroz.

\section{Respiración de la biomasa microbiana del suelo "RBM"}

Los cambios observados en la RBM del suelo en tres etapas del ciclo de desarrollo del arroz se presentan en la Figura 4. La respiración del suelo corresponde a la producción de $\mathrm{CO}_{2}$ por el metabolismo de los microorganismos que viven dentro de la matriz del suelo (Anderson 1982). Aunque en LMC la respiración presentó valores levemente mayores a los de SDR, no hubo diferencias estadísticamente significativas entre sistemas de labranza. En ambos casos se observó una tendencia creciente en la tasa de respiración, entre la

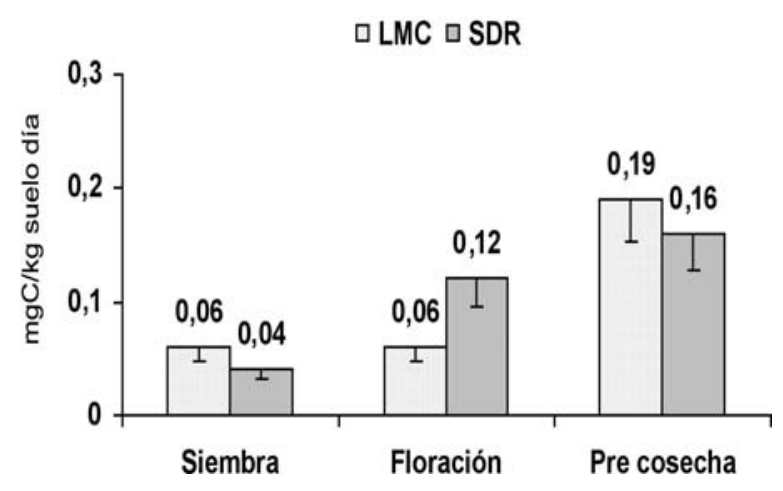

Figura 4. Respiración de la biomasa microbiana "RBM" del suelo en tres diferentes fases del ciclo de desarrollo de un arrozal inundado. Lote Playitas, Guanacaste, Costa Rica. 2004.

siembra y antes de la cosecha del arroz ésta pasó de 0,05 a más de $0,18 \mathrm{mgC} / \mathrm{kg}$ suelo día. Esto corresponde al período inundado del cultivo y por ende posiblemente refleje la actividad de los microorganismos anaeróbicos. Sin embargo, en el análisis anterior debe tenerse presente que los resultados son producto de mediciones de la respiración de microorganismos aeróbicos o anaeróbicos facultativos que estaban presentes en el suelo, luego de tomadas las muestras y durante el período de análisis. Estos resultados no permiten realizar un análisis in situ ni miden la actividad de los organismos anaeróbicos del agroecosistema inundado.

\section{CONCLUSIÓN}

En el presente estudio, la labranza del terreno y la incorporación de los rastrojos de arroz en LMC afectaron en forma diferente la cantidad de NBM con respecto a cuando los rastrojos se dejaron sobre la superficie del terreno en un suelo sin laborar conforme al sistema SDR. En todos los casos la cantidad de NBM en los primeros $5 \mathrm{~cm}$ del perfil fue mayor en SDR. En este sistema, la siembra de mucuna entre dos ciclos de cultivo de arroz, aportó rastrojos con baja relación $\mathrm{C}: \mathrm{N}$, que permitió en combinación con los rastrojos de arroz acumulados de ciclos previos y en distinto grado de descomposición, una mayor concentración de MOS, la cual pasó de 2,24\% a 4,1\% de un ciclo de cultivo al siguiente. El NBM también incrementó significativamente, y aumentó cuatro veces la cantidad existente al inicio del ciclo previo. 
Debido a la condición anaeróbica producida por la cobertura con lámina de agua del suelo durante la mayor parte del desarrollo del cultivo, RBM del suelo y la mineralización del $\mathrm{N}$ fueron restringidas. $\mathrm{La}$ RBM mostró una tendencia creciente conforme se desarrolló el cultivo, y se observó un aumento en la actividad microbiana. La actividad de la biomasa microbiana del suelo puede ser aún mayor entre la cosecha y el inicio de la siembra del cultivo siguiente, cuando en ausencia de una lámina de agua predominan condiciones aeróbicas en el suelo. Realizar prácticas de manejo que permitan maximizar la contribución de los rastrojos como fuente de nutrimientos para el cultivo durante dicho lapso, debería ser una parte importante de los programas de manejo integrado de agroecosistemas inundados.

Debido a que los microorganismos del suelo pueden controlar la tasa a la cual el $\mathrm{N}$ está disponible para la absorción del cultivo, y si se considera que éstos al inmovilizar, retener, e impedir la rápida transformación del nutrimento, limitan significativamente sus pérdidas por lixiviación o volatilización; por propiciar mayor actividad de estos organismos vivientes, el sistema SDR es más apropiado para aprovechar los aportes de nutrimientos de la MOS y reducir la fertilización nitrogenada durante el cultivo.

\section{LITERATURA CITADA}

ANDERS, M.; OLK, D.; HARPER, T.; DANIEL, T.; HOLZHAUER J. 2004. The effect of rotation, tillage and fertility on rice grain yields and nutrient flows. In: Proc. $26^{\text {th }}$ Southern Conservation Tillage Conference. p. 26-29.

ANDERSON, J. 1982. Soil respiration. In: Page, A.L. ed. Methods of soil analysis: Part 2. Chemical and microbiological properties. ASA. Agron. Monogr. 9. Madison. p. 831-866.

ANDERSON, T.; DOMSCH, K.H. 1989. Ratio of microbial biomass Carbon to total biomass organic carbon. In: Arable soils. Soil Biol. Biochem. 21: 471-479.

ANGERS, D.; RECOUS, S. 1997. Decomposition of wheat straw and rye residues as affected by particle size. Plant and Soil. 189: 197-203.
BAYER, C.; MARTIN-NETO, L.; MIELNICZUK, J.; PILLON, C.N.; SANGOI, L. 2001. Changes in Soil Organic Matter Fractions under Subtropical No-Till Cropping Systems. Soil Sci. Soc. Am. J. 65: 1473-1478.

BERTSH, F. 2003. Abonos orgánicos, manejo de la fracción orgánica y de los aspectos biológicos del suelo. In: Meléndez G.; Molina E. (eds). Fertilizantes: características y manejo. Centro de Investigaciones Agronómicas, Universidad de Costa Rica. p. 117-135.

BREMER, E.; KESSEL, C. VAN 1992. Plant-available nitrogen from lentil and wheat residues during a subsequent growing season. Soil Science Society of America Journal. 56:1155-1160.

CABRERA, M.L.; VIGIL, M.F.; KISSEL, D.E. 1994. Potential Nitrogen Mineralization: Laboratory and field evaluation. In: Hablin (eds). Soil Testing: Prospect for improving nutrient recommendation. p. 15-30.

CARTER, M.; GREGORICH, E.; ANGERS, R.; DONALD, R.; BOLINDER, M. 1998. Organic C and N storage, and Organic $\mathrm{C}$ fractions, in adjacent cultivated and forested soils of eastern Canada. Soil Till. Res. 47:253-261.

CASSMAN, K.; DE DATTA, S.; OLG, D.; ALCÁNTARA, J.; SAMSON, J.; DESCALSOTA, J.; DIZON, M. 1995. Yield decline and the nitrogen economy of longterm experiments on continuous, irrigated rice systems in the tropics. In: Lal and B. A. Stewart eds. Soil management experimental basis for sustainability and environmental quality. R. CRC Lewis Publishers. Boca Raton, Fla. p. 181-222.

CASTRO, L. 1995. Efecto del uso agrícola y el barbecho sobre los contenidos de biomasa microbiana de ultisoles y andisoles de Costa Rica. Agronomía Costarricense 19(2):59-65.

DORAN, J. 1980. Soil microbial and biochemical changes associated with reduced tillage. Soil Sci. Soc. Am. J. 44:765-771.

DORAN, J.; PARKIN, T. 1994. Defining and assessing soil quality. In: Doran J.W.; Coleman D.C.; Bezdicek D.F.; Stewart B.A. eds. Defining soil quality for a sustainable environment. Soil Sci. Soc. Am., Am. Soc. Agron., Madison, WI. p 3-21. 
ETANA, A.; HAKANSSON, I.; ZAGAL E.; BUCAS, S. 1999. Effects of tillage dept on organic carbon content and physical properties in five Swedish soils. Soil \& Tillage Research. 52, 129-139.

FERRERA, R.; ALARCÓN, A. 2001. La microbiología del suelo en la agricultura sostenible. Ciencias Naturales y Agropecuarias, México. 8: 175-183.

FORNASIER, F.; MONDINI, C.; LEITA, L. 2002. Dynamics of soil microbioal biomass and organic matter quality following adition of pig slurries. In: J. Venglovsk_and G. Gréserová. eds. Recyclin of Agricultural, Municipal and Industrial Residues in Agricultural. Proceedings of the $19^{\text {th }}$ International Council of the Ramiran Network. p. 473-476.

FRANZLUEBBER, S. J. 2002. Soil organic matter stratification ratio as an indicator of soil quality. Soil \& Tillage Research. 66: 95-106.

FRUIT, L. 1999. Organic matter decomposition: effect of soil porosity and plant residue particle size. In: Berthelin J.; Huang P.M.; Bollag J.M.; Andreux F. eds. Effect of Mineral-Organic-Microorganism Interactions on Soil and Freshwater Environments. Plenum Publishing Corporation. New York. p. 189-196.

GALE, W.; CAMBARDELLA, C. 2000. Carbon dynamics of surface residue- and root-derived organic matter under simolated No-Till. Soil Sci Soc. Am. J. 64: 190-195.

GONZÁLEZ, M.; RAMÍREZ, C. 1991. Respuesta del arroz (Oryza sativa) a la fertilización nitrogenada en rotación con sorgo (Sorghum bicolor) y soya (Glycine max). Agronomía Costarricense 15: 143-149.

HARRIS, G.; HESTERMAN, O. 1990. Quantifying the nitrogen contribution from alfalfa to soil and two succeeding crop using nitrogen-15. Agronomy Journal 82: $129-134$

HORWATH, W.; KESSEL, C. 1998. Reassessing soil N availability and fertilizer recommendations under alternative rice residue management practices (En línea). Consultado 12 nov. 2004. Disponible en: http://www.syix.com/rrb/98rpt/Soil\%20N.htm

IRRI (International Rice Research Institute). 1990. IRRI Beefs up biological nitrogen fixation. (En línea).
Consultado 14 nov. 2004. Disponible en: http://fadr.msu.ru/rodale/agsieve/txt/vol3/3/a2.html

JANZEN, H.; SCHAALJE, G. 1992. Barley response to nitrogen and non nutritional benefits of legume green manure. Plant and Soil. 142:19-30.

JOHNSON, A. 1991. Potential changes in soil fertility from arable farming, including organic systems. Soils and Agronomy Department, AFRC Institute of Arable Crops Research, Rothamsted Experimental Station, Harpenden, United Kingdom. 38 p.

LADHA, J.K.; DAWE, D.; VENTURA, T.S.; SINGH, U.; VENTURA, W.; WATANABE, I. 2000. Long-term effects of urea and green manure on rice yields and nitrogen balance. Soil Sci. Soc. Am. J. 64:1993-2001.

LEITA, L.; DE NOBILI, M.; MONDINI, C.; MUHLPABACHOVA, G.; MARCHIOL, L.; BRAGATO, G.; CONTIN, M. 1999. Influence of inorganic and organic fertilization on soil microbial biomass, metabolic quotient and heavy metal bioability. Biol. Fertil. Soils. 28: 371-376.

LUNDQUIST, E.; SCOW, K.; JACKSON, L.; UESUGI, S.; JOHNSON, C. 1999. Rapid response of soil microbial communities from conventional, low input and organic farming systems to a wet/dry cycle. Soil Biol. Biochem. 31: 1661-1675.

MELÉNDEZ, G. 1997. Transformaciones del carbono, nitrógeno y fósforo del suelo, en sistemas agroforestales, cultivos anuales y bosque natural. Tesis M.Sc San José, Universidad de Costa Rica. 105 p.

NORMAN, R.; WILSON, C.; SLATON, N. 2003. Soil fertilization and mineral nutrition in U.S. mechanized rice culture. In: Smitn C.W.; Dilday R.H. (eds). Rice, Origin, History, Technology and Production. p. 331-413.

PALANIAPPAN, S.P.; SIDDESWARAN, K. 1999. Regional overview on green manure in rice-based croppins systems. Nagarjuna Agricultural Research and Development Institute. Andhra Pradesh, India. p. 126-135.

POWLSON, D.; BROOKES, P. 1987. Measurement of soil microbial biomass provides an early indication of changes on total soil organic matter due to straw incorporation. Soil. Biochem. 19: 159-164.

RASMUSSEN, P.; COLLINS, H. 1991. Long-term impacts of tillage, fertilizer and crop residue on soil organic 
matterr in temperate semiarid regions. Adv. Agron. 45: 93-134.

REEVES, D. 1997. The role of soil organic matter in maintaining soil quality in continuous cropping systems. Soil Tillage Res. 43: 131-167.

SALAS, E.; RAMÍREZ, C. 1999. Bioensayo microbiano para estimar los nutrimentos en los abonos orgánicos: calibración en el campo. In: Bertsh, F. et al. ed. Memoria Congreso Agronómico Nacional y III Congreso Nacional de Suelos. Memorias, Vol 3, Recursos Naturales y Producción Animal. Costa Rica. p. 71.

SHELTON, D.; JASA, P.; BROWN, L.; HIRSCHI, M. 2000. Water Erosion. In: Conservation Tillage, Systems and Management. MidWest Plan Service MWPS-45. Washington. p. 19-28.

SINGH, B.M.; SRIVASTAVA, V.K.; KIDWAI, M.A.; GUPTA, V.; GUPTA, R. 1995. Aloe, psoralea and mucuna. In: Adv. in horticulture Vol 11. Medicinal and aromatic plants. Malhotra Publ, House, New Delhi. p. 512-525.

THENG, B. 1987. Clay-humic interactions and soil aggregate stability. In: Rengasamy, P. ed. Soil Structure and Aggregate Stability. Institute of Irrigation and Salinity Research, Tatura. India. p. 32-73.
TIESSEN, H.; CUEVAS, E.; CHACON, P. 1994. The role of soil organic matter stability in soil fertility and agricultural potential. Nature 371: 783-785.

URIBE, L. 1999. Uso de indicadores microbiológicos de suelos: ventajas y limitaciones. In: Bertsch et al. ed. Memoria XI Congreso Nacional Agronómico y de Recursos Naturales, Vol. 3, Recursos Naturales y Producción Animal. San José. p. 39-46.

VORONEY, R.; PAUL, E.; ANDERSON, D. 1989. Decomposition of wheat straw and stabilization of microbial products. Can J. Soil Sci. 69: 63-77.

WARDLE, D. 1992. Comparative asessment of factors wich influence microbial biomass carbon and nitrogen levels in soil. Biol. Rev. 67:321-358.

YAMAGUCHI, M. 1979. Biological nitrogen fixation in floodeed rice field. In: Nitrogen and rice. International Rice Research Institute (IRRI). Philippines. p. $192-203$.

ZAK, D.; TILMAN, D.; PARMENTER, R.; RICE, C.; FISHER, F.; VOSE, J.; MILCHUNAS, D.; MARTIN, C. 1994. Plant production and soil microorganisms in late successional ecosystems: a continental scale study. Ecology 75(8): 2333-2347. 